\title{
A Polarimetric Extension of the van Cittert-Zernike Theorem for Use with Microwave Interferometers
}

\author{
J.R. Piepmeier, Member, IEEE, and N.K. Simon
}

\begin{abstract}
The van Cittert-Zernike theorem describes the Fourier-transform relationship between an extended source and its visibility function. Developments in classical optics texts use scalar field formulations for the theorem. Here, we develop a polarimetric ertension to the van Cittert-Zernike theorem with applications to passive microwave Earth remote sensing. The development provides insight into the mechanics of twodimensional inteferometric imaging, particularly the effects of polarization basis differences between the scene and the observer.
\end{abstract}

Index Terms-Interferometer, polarization, polarimetric, STAR

\section{INTRODUCTION}

$\mathbf{T}$ HE desire to obtain fine spatial and temporal resolution passive microwave imagery of Earth from space drives the need for microwave instruments with large apertures. As aperture size increases beyond current practice, however, imaging by mechanical scanning becomes challenging because of structural dynamics. Electronic scanning methods, on the other hand, alleviate the need to move large antenna structures. One such electronic method is microwave interferometry, also called synthetic thinned array radiometry (STAR) [1]. An interferometer measures the complex spatial correlation field, or visibility function, of the incident radiation originating from a source. The van Cittert-Zernike theorem describes the Fourier transform relationship between a source distribution and its associated visibility function. In classical optics texts, the van Cittert-Zernike theorem is developed using scalar fields (see e.y., [2], [3]). Likewisise, tu dâtu the only cxporimentally demonstrated STAR instruments have been single polarization and can be described by the scalar theory (see e.g., [4]). An interesting effect occurs in two-dimensional (2-D) STAR imaging: the observer and source polarization basis coordinates do not match in all directions of observation. Thus, the polarization basis can be rotated differently for each pixel in an image. This indeed is recognized by the developers of the 2-D STAR instrument on the Soil Moisture Ocean Salinity (SMOS) mission [5]. Here, the polarimetric extension to the van Cittert-Zernike theorem lends particular insight into this process.

Our development follows the form of [2] with the addition of partially polarized radiation from the extended source. Another recent development included partially polarized light

This work was supported by the NASA Earth Science New Investigator Program.

J.R. Piepmeier is with NASA's Goddard Space Flight Center. N.K. Simon was with QSS Group, Inc.and is now with the International Spy Museum, Washington, D.C.

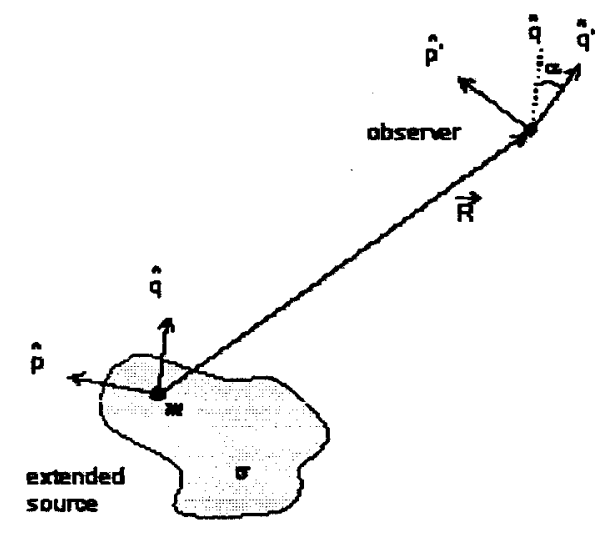

Fig. 1. Infintesimal source $m$ within the extended source $\sigma$ radiates and is observed at the far-zone location $\vec{R}$. The observer polarization basis is rotated by $\alpha$ with respect to the source.

[6]; however, the analysis used the paraxial approximation for propagation in beam optics. Here, as in [2], we apply the spherical wave approximation appropriate for far-field propagation of waves originating from infinitesimal point sources. The polarimetric visibility function is expressed using the so-called coherency vector [7], which contains the same information as the mutual coherency matrix used in [5] and the bearn coherence-polarization matrix used in [6]. The cherency vector can be transformed to the modified Stokes visibility vector, common in radio-astronomical polarimetry [8], by a simple matrix operator. Additionally, we consider the differing polarization basis coordinates of the source and the observer. Polarization rotation of the electric fields is performed using Jones matricies. (For a formal description relating Jones matricies to the Stokes vector see [9].) Our result is instructive for interpreting 2-D STAR imagery of the Earth.

\section{RADIATED FIELD}

The far-zone electric field $\bar{E}_{m}$ at location $\vec{R}$ arising from an infinitesimal element $m$ within the extended source $\sigma$ can be written (see Fig.1):

$$
\bar{E}_{m}(\overrightarrow{\mathrm{R}}, t)=\bar{A}_{m}\left(\widehat{k}, t-\frac{R}{c}\right) \frac{e^{-j 2 \pi \frac{\int}{c} \vec{k} \cdot \vec{k}}}{R}
$$

where $R=|\vec{R}|$ is the range, $\hat{k}$ is the direction of propa.0 of light, and $f$ is the frequency. This 
expression is the vector phasor representation of a partiallypolarized quasi-monochromatic field with complex envelope $\bar{A}_{m}$. The complex vector random process $\bar{A}_{m}$ contains both the direction and polarization dependence of the radiation emitted by the surface element and is assumed to be zero mean and stationary. The electric field is related to the brightness distribution due to $m$ by [10]

$$
B_{m}(\hat{\mathrm{k}}) d \Omega=\frac{1}{\eta_{0}}\left\langle\left|\bar{E}_{m}(\overrightarrow{\mathrm{R}})\right|^{2}\right\rangle
$$

where $\eta_{0}$ is the intrinsic impedance of free space and $d \Omega$ is a differential solid angle. Its envelope is similarly related by

$$
B_{m}(\hat{k}) d \sigma=\frac{1}{\eta_{0}}\left\langle\left|\bar{A}_{m}(\hat{k})\right|^{2}\right\rangle
$$

where $d \sigma=d \Omega / R^{2}$ is differential surface area. Note, the above expression is the radiation intensity or pattern of the infinitesimal source.

The electric field and its envelope have two polarization components in directions $\widehat{p}$ and $\widehat{q}$, the polarization basis vectors of the source frame:

$$
\bar{E}_{m}=\left[\begin{array}{c}
E_{m, p} \\
E_{m, q}
\end{array}\right], \quad \bar{A}_{m}=\left[\begin{array}{c}
A_{m, p} \\
A_{m, q}
\end{array}\right] \text {. }
$$

The polarization basis vectors form a right-handed Cartesian basis with $\widehat{\mathrm{k}}$ such that $\widehat{\mathrm{k}}=\widehat{\mathrm{p}} \times \widehat{\mathrm{q}}$. The process $\bar{A}_{m}$ is related to the modified Stokes vector $\bar{I}_{\sigma}(\hat{\mathrm{k}})$ of the source in the $\widehat{\mathrm{p}}-\widehat{\mathrm{q}}$ polarization basis:

$$
\bar{I}_{\sigma}(\widehat{\mathrm{k}}) d \sigma=\left[\begin{array}{c}
I_{p} \\
I_{q} \\
U \\
V
\end{array}\right] d \sigma=\frac{1}{\eta_{0}}\left[\begin{array}{c}
\left\langle\left|A_{m, p}(\hat{\mathrm{k}})\right|^{2}\right\rangle \\
\left\langle\left|A_{m, q}(\widehat{\mathrm{k}})\right|^{2}\right\rangle \\
2 \operatorname{Re}\left\langle A_{m, p}(\hat{\mathrm{k}}) A_{m, q}^{*}(\hat{\mathrm{k}})\right\rangle \\
2 \operatorname{Im}\left\langle A_{m, p}(\hat{\mathrm{k}}) A_{m, q}^{*}(\hat{\mathrm{k}})_{5}\right\rangle
\end{array}\right]
$$

where the subscript $\sigma$ is used to denote the continuous dependence upon location within the source [10].

The polarization basis vectors in the observation frame $\widehat{\mathrm{p}}^{\prime}$ and $\widetilde{q}^{\prime}$ and the feld in the chserration fame $\vec{E}_{m}^{\prime}$ are related to the source frame by a Jones matrix of polarization basis rotation:

$$
\overline{\bar{P}}(\alpha)=\left[\begin{array}{ll}
\widehat{\mathrm{p}} \cdot \widehat{\mathrm{p}}^{\prime} & \widehat{\mathrm{q}} \cdot \widehat{\mathrm{p}}^{\prime} \\
\widehat{\mathrm{p}} \cdot \widehat{\mathrm{q}}^{\prime} & \widehat{\mathrm{q}} \cdot \widehat{\mathrm{q}}^{\prime}
\end{array}\right]=\left[\begin{array}{cc}
\cos \alpha & \sin \alpha \\
-\sin \alpha & \cos \alpha
\end{array}\right]
$$

such that

$$
\bar{E}_{m}^{\prime}=\overline{\bar{P}}(\alpha) \bar{E}_{m}, \quad \bar{A}_{m}^{\prime}=\overline{\bar{P}}(\alpha) \bar{A}_{m}
$$

where $\alpha$ is the angle of polarization basis rotation (PBR):

$$
\alpha=\tan ^{-1} \frac{\widehat{q} \cdot \widehat{p}^{\prime}}{\widehat{q} \cdot \widehat{q}^{\prime}} \text {. }
$$

The total electric field received by an isotrope is found by summing over all elements within the extended source:

$$
\begin{aligned}
\vec{E}^{\prime}(\overrightarrow{\mathrm{R}}, t)= & \sum_{m \in \sigma} \overline{\bar{P}}\left(\alpha_{m}\right) \bar{A}_{m}\left(\widehat{\mathrm{k}}_{m}, t-\frac{R_{m}}{c}\right) \\
& \frac{e^{-j 2 \pi \frac{f}{c} \widehat{\mathrm{k}}_{m} \cdot \overrightarrow{\mathrm{R}}_{m}}}{R_{m}}
\end{aligned}
$$

\section{VISIBILITY FUNCTION}

The coherency vector of electric fields received by two isotropes with displacement $\vec{D}$ is defined by [7]:

$$
\bar{V}(\overrightarrow{\mathrm{D}})=\left\langle\bar{E}^{\prime}(\overrightarrow{\mathrm{R}}, t) \otimes \bar{E}^{\prime *}(\overrightarrow{\mathrm{R}}+\overrightarrow{\mathrm{D}}, t)\right\rangle
$$

where $\otimes$ is the outer product operator. Use of the coherency vector to represent the visibility function is convenient because its elements relate directly to the co-polarized and crosspolarized complex correlations measured with an interferometer. The outer product is expanded to yield

$$
\bar{V}(\overrightarrow{\mathrm{D}})=\left[\begin{array}{l}
\left\langle E_{p}^{\prime}(\overrightarrow{\mathrm{R}}) E_{p}^{\prime *}(\overrightarrow{\mathrm{R}}+\overrightarrow{\mathrm{D}})\right. \\
E_{p}^{\prime}(\overrightarrow{\mathrm{R}}) \\
E_{q}^{\prime *}(\overrightarrow{\mathrm{R}}+\overrightarrow{\mathrm{D}}) \\
E_{q}^{\prime}(\overrightarrow{\mathrm{R}}) \\
E_{p}^{\prime *}(\overrightarrow{\mathrm{R}}+\overrightarrow{\mathrm{D}}) \\
E_{q}^{\prime}(\overrightarrow{\mathrm{R}}) E_{q}^{\prime *}(\overrightarrow{\mathrm{R}}+\overrightarrow{\mathrm{D}})
\end{array}\right\} .
$$

Substituting (9) into (10) and using the parallel-ray approximation, we obtain

$$
\begin{aligned}
\bar{V}(\overrightarrow{\mathrm{D}})= & \sum_{m \in \sigma}\left\langle\left\langle\overline{\bar{P}}\left(\alpha_{m}\right) \bar{A}_{m}\left(\widehat{\mathrm{k}}_{m}, t-\frac{|\overrightarrow{\mathrm{R}}|}{c}\right)\right.\right. \\
& \left.\otimes \overline{\bar{P}}\left(\alpha_{m}\right) \bar{A}_{m}^{*}\left(\widehat{\mathrm{k}}_{m}, t-\frac{|\overrightarrow{\mathrm{R}}+\overrightarrow{\mathrm{D}}|}{c}\right)\right\rangle \\
& \cdot \frac{e^{j 2 \pi \hat{c}_{m} \widehat{\mathrm{k}}_{m} \cdot \overrightarrow{\mathrm{D}}}}{|\overrightarrow{\mathrm{R}}||\overrightarrow{\mathrm{R}}+\overrightarrow{\mathrm{D}}|}
\end{aligned}
$$

A property from tensor algebra allows some rearranging:

$$
\begin{aligned}
\bar{V}(\overrightarrow{\mathrm{D}})= & \sum_{m \in \sigma}\left(\overline{\bar{P}}\left(\alpha_{m}\right) \otimes \overline{\bar{P}}\left(\alpha_{m}\right)\right) \\
& \cdot\left\langle\bar{A}_{m}\left(\widehat{\mathrm{k}}_{m}\right) \otimes \bar{A}_{m}^{*}\left(\widehat{\mathrm{k}}_{m}\right)\right\rangle \frac{e^{j 2 \pi \frac{f}{c} \widehat{\mathrm{k}}_{m} \cdot \overrightarrow{\mathrm{D}}}}{|\overrightarrow{\mathrm{R}}||\overrightarrow{\mathrm{R}}+\overrightarrow{\mathrm{D}}|}
\end{aligned}
$$

The time differences in the expected value are neglected because $\bar{A}_{m}$ is assumed to remain self-coherent at a time lag of $\bar{D} / c$. Examining the expected vaiue, we nind

$$
\left\langle\bar{A}_{m}\left(\hat{\mathrm{k}}_{m}\right) \otimes \bar{A}_{m}^{*}\left(\hat{\mathrm{k}}_{m}\right)\right\rangle=\eta_{0} \overline{\bar{S}} \bar{I}_{\sigma}\left(\hat{\mathrm{k}}_{m}\right) d \sigma
$$

where

$$
\overline{\bar{S}}=\left[\begin{array}{cccc}
1 & 0 & 0 & 0 \\
0 & 0 & \frac{1}{2} & \frac{j}{2} \\
0 & 0 & \frac{1}{2} & -\frac{j}{2} \\
0 & 1 & 0 & 0
\end{array}\right]
$$

transforms the modified Stokes vector (5) to a coherency vector representation [7]. The coherency vector can now be rewritten as an integral over the source

$$
\bar{V}(\overrightarrow{\mathrm{D}})=\eta_{0} \int_{\sigma}(\overline{\bar{P}}(\alpha) \otimes \overline{\bar{P}}(\alpha)) \overline{\bar{S}} \bar{I}_{\sigma}(\hat{\mathrm{k}}) \frac{e^{j 2 \pi \frac{f}{c} \hat{\mathrm{k}} \cdot \overrightarrow{\mathrm{D}}}}{R_{1} R_{2}} d \sigma
$$

or over solid angle

$$
\bar{V}(\overrightarrow{\mathrm{D}})=\eta_{0} \int_{4 \pi}(\overline{\bar{P}}(\alpha) \otimes \overline{\bar{P}}(\alpha)) \overline{\bar{S}} \bar{I}_{\sigma}(\widehat{\mathrm{k}}) e^{j 2 \pi \frac{\delta}{c} \hat{\mathrm{k}} \cdot \overrightarrow{\mathrm{D}}} d \Omega
$$


The outer product $\overline{\bar{P}}(\alpha) \otimes \overline{\bar{P}}(\alpha)$ acts as a PBR matrix in coherency vector space. Equation 17 can be written with the familiar PBR matrix for the modified Stokes vector [11]

$$
\bar{V}(\overrightarrow{\mathrm{D}})=\eta_{0} \int_{4 \pi} \overline{\bar{S}} \overline{\bar{R}}(\alpha) \bar{I}_{\sigma}(\hat{\mathrm{k}}) e^{j 2 \pi \frac{I}{\mathrm{c}} \cdot \overrightarrow{\mathrm{D}}} d \Omega
$$

where

$$
\overline{\bar{R}}(\alpha)=\left[\begin{array}{cccc}
\cos ^{2} \alpha & \sin ^{2} \alpha & \frac{1}{2} \sin 2 \alpha & 0 \\
\sin ^{2} \alpha & \cos ^{2} \alpha & -\frac{1}{2} \sin 2 \alpha & 0 \\
-\sin 2 \alpha & \sin 2 \alpha & \cos 2 \alpha & 0 \\
0 & 0 & 0 & 1
\end{array}\right] .
$$

The expression in (18) is the polarimetric extension of the classical van Cittert-Zernike theorem.

\section{Application to Earth Remote SEnsing}

We can consider the visibility function and the brightness distribution to be a Fourier-transform pair described by (18):

$$
\bar{V}(\overrightarrow{\mathrm{D}}) \leftrightharpoons \eta_{0} \overline{\bar{S}} \bar{I}_{\sigma}^{\prime}(\hat{\mathrm{k}})
$$

where

$$
\bar{I}_{\sigma}^{\prime}(\widehat{\mathrm{k}})=\overline{\bar{R}}(\alpha) \bar{I}_{\sigma}(\widehat{\mathrm{k}})
$$

is the brightness distribution in the observer polarization basis. Thus, the key difference between the polarimetric and scalar formulations of the van Cittert-Zernike theorem, besides (18) being in vector form, is the polarization basis rotation between the source and observer. This variation impacts the interpretation of 2-D STAR imagery of the Earth. For example, when the measured visibility function is inverted to obtain an image, each pixel in the reconstructed image will have a polarization basis different from the Earth. To obtain the brightness distribution in the Earth's polarization basis, the inverse of the polarization basis rotation matrix is applied for each observation direction:

$$
\bar{I}_{\sigma}(\hat{\mathrm{k}})=\overline{\bar{R}}^{-1}(\alpha) \bar{I}_{\sigma}^{\prime}(\hat{\mathrm{k}}) \text {. }
$$

Here, we consider the case of a nadir-viewing 2-D STAR and will determine the polarization basis rotation angle across the image. We define the Earth's natural polarization basis such thiat the hurizoniai poiarization $\widehat{h}_{e}$ is parallel to the surface and the vertical polarization is $\widehat{v}_{e}=\widehat{h}_{e} \times \widehat{k}$ with $\widehat{k}$ pointing away from the surface. As shown in Fig.2, the origin of the observer coordinate frame is placed at height $H$ above the surface with $\widehat{z}$ pointing down and $\hat{k}=-\widehat{r}$.The source polarization basis is aligned with Earth's natural polarization basis, which yields

$$
\widehat{\mathrm{p}}=\widehat{\mathrm{v}}_{e}=\widehat{\theta} \quad \widehat{\mathrm{q}}=\widehat{\mathrm{h}}_{e}=-\widehat{\phi} .
$$

The observer polarization basis is arbitrarily aligned to obtain $\widehat{\mathrm{p}}^{\prime}=\widehat{x}$ at nadir $(\theta=\phi=0)$. The conventional Ludwig's third definition for polarization basis of an antenna is used to define [12]

$$
\begin{array}{lr}
\widehat{p}^{\prime}= & \cos \phi \widehat{\theta}-\sin \phi \widehat{\phi} \\
\widehat{q}^{\prime}= & -\sin \phi \widehat{\theta}-\cos \phi \widehat{\phi}
\end{array}
$$

Finally, the polarization basis rotation angle for a pixel in direction $(\theta, \phi)$ is found by substituting (23-24) into (8):

$$
\alpha=\phi \text {. }
$$

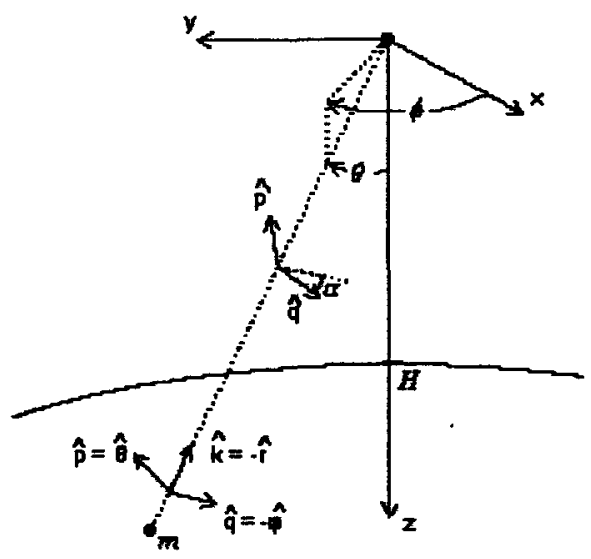

Fig. 2. With the observer coordinate frame located at $H$ above the Earth, the polarization basis is rotated by $\alpha$ for pixel $m$ located in the $(\theta, \phi)$ direction.

Thus, the polarization basis in a single image channel will rotate with the azimuth angle in the image. For example, the $I_{p}^{\prime}$ image will contain a mixture of $I_{v}, I_{h}$, and $U$ depending upon the azimuth angle. With $\phi=0, I_{p}^{\prime}=I_{v}$, but with $\phi=90^{\circ}, I_{p}^{\prime}=I_{h}$. Thus, to obtain the $I_{h}$ image, for example, the entire $\vec{I}^{\prime}$ vector is needed.

\section{DISCUSSION}

Here, we developed the polarimetric version of the van Cittert-Zernike theorem, which describes the Fourier transform relationship of a brightness distribution with its visibility function. Using the coherency vector to represent the polarimetric visibility function yields a compact expression that provides insight into interferometer operation. For example, this expression demonstrates how polarization basis differences between source and observer affect a reconstructed image. When imagery is desired in the Earth's natural polarization basis, the full complex coherency vector needs to be measiited. Designers, however, may be tempted not to measure crosspolarization correlations to save engineering resources. If only the two co-polarized visibilities are measured, then PBR cannot be completely removed. Mathematically, $\overline{\bar{R}}$ becomes a $2 \times 2$ matrix that is ill-conditioned near $\pm 45^{\circ}$, at which it becomes singular. On the other hand, imagery in the instrument's polarization basis may be acceptable. In atmospheric sounding, legacy real-aperture instruments already exhibit varying polarization basis. Thus, instrumental PBR, as described by the polarimetric van Cittert-Zernike theorem, could be incorporated into the retrieval of atmospheric parameters from interferometer imagery.

\section{REFERENCES}

[1] D. M. Le Vine, "Synthetic aperture radiometer systems," IEEE Trans. Geosci. Remote Sensing, vol. 37, no. 12, pp. 2228-2236, 1999.

[2] M. Born and E. Wolf, Principles of Optics, 7th ed. New York: Cambridge University Press, 1999.

[3] J. W. Goodman, Statistical Optics. New York: Wiley, 1985. 
[4] C. S. Ruf, C. T. Swift, A. B. Tanner, and D. M. Le Vine, "Interferometric synthetic aperture microwave radiometry for the remote sensing of the Earth," IEEE Trans. Geosci. Remote Sensing, vol. 26, no. 5, pp. 597$611,1988$.

[5] M. Martin-Neira, S. Ribo, and A. J. Martin-Polegre, "Polarimetric mode of MIRAS," IEEE Trans. Geosci. Remote Sensing, vol. 40, no. 8, pp. 1755-1768, 2002.

[6] F. Gori, M. Santarsiero, R. Borghi, and G. Piquero, "Use of the van Cittert-Zernike theorem for partially polarized sources," Optics Letters, vol. 25, no. 17, pp. 1291-1293, 2000.

[7] J. P. Hamaker, J. D. Bregman, and R. J. Sault, "Understanding radio polarimetry 1. Mathematical foundations," Astronomy and Astrophysics Supplement Series, vol. 117, no. 1, pp. 137-147, 1996.

[8] A. R. Thompson, J. M. Morgan, and G. W. Swenson, Jr., Interferometry and Synthesis in Radio Astronomy. Malabar, Florida: Krieger Publishing Company, 1991.

[9] R. Schmeider, "Stokes algebra formalism," "J. Opt. Soc. Amer.", vol. 59, pp. 297-302, 1969.

[10] A. K. Fung Microwave Scattering and Emission Models and Their Applications. Boston: Artech House, 1994

[11] S. Chandrasekhar, Radiative Transfer. New York: Dover Publications, 1960.

[12] A. C. Ludwig, "The definition of cross polarization," IEEE Trans. Antennas Prop., vol. AP-21, pp. 197-200, Jan. 1973. 